\title{
Usine marémotrice de La Rance : reconstruction des alternateurs
}

par J. Salvi

SARELEM

\section{LE GROUPE BULBE}

Queiques rappels simples

C'est un ensemble électro-mécátrique qui rassemble dans une même coguc, ou enceinte ćtanchc, inmergće dans un conduit hydraulicque, tous les ćlćments nécessaires à la production d'énergic

- alternateur avec ses auxiliaires.

- paliers permettant la rotation et le guidage axial,

- accouplements mécaniques, etc..

La forme donnée à l'enceinte, pour tavoriser l'écoulement d'eau, rappelle celle d'un bulbe de plante, d'où le nom donné à ce type de groupe.

L'énergie hydraulique est captée par la turbine, placéc en dehors de l'enceinte, qui entraince en rotation l'cnsemble de la partie tournante du groupe.

Cette turbine est de type Kaplan à axe horizontal et à pales orientables qui permettent d'ajuster la puissance hydratiligue motrice à la demande électrique.

Le groupe bulbe est un générateur de type «basse chute " don l'énergie hydraulique est surtout produite par te débit ct peu par la hauteur. Sa fréquence de rotation est basse, de l'ordie de queloues $\mathrm{H} z$.

\section{II — LES GROUPES BULBES DE L'USINE MARÉMOTRICE DE LA RANCE}

L'usine marémourice de la Rance comporte 24 groupes bulbes de puissance unitaire $10 \mathrm{MVA}$ soit $240 \mathrm{MVA}$ installés.

Ces 24 groupes constituent 6 unités d'exploitation.

E'unité d'exploitation est donc formée d'un «ensemble " de 4 groupes qui fonctionnent simultanément et disposent en commun d'un certain nombre d'organes annexes notamment pour le réglage des turbines et l'excitation des afternateurs.

Chaque urite diexploitation, ou ensemble, débite sur l'un

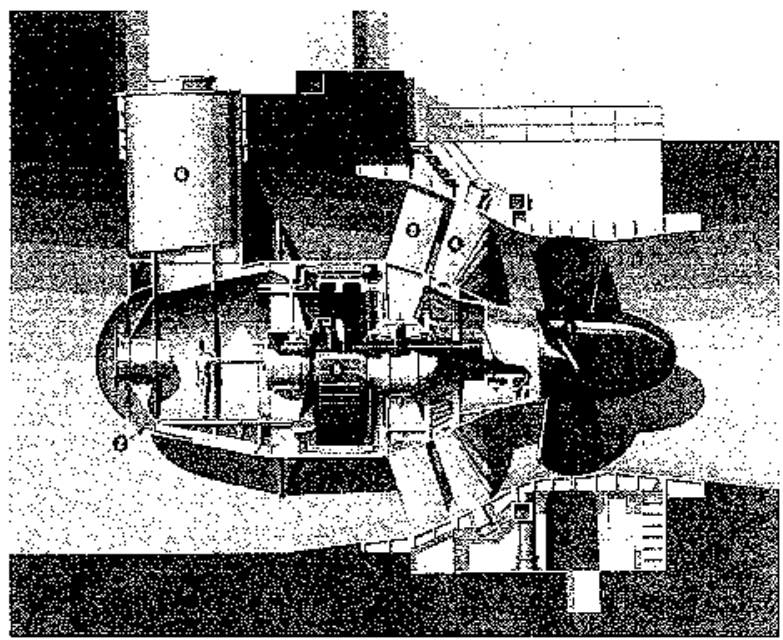

Le groupe bulbe de la Rance

- Roue motrice : diamètre $5,35 \mathrm{~m}$, vitesse $94 \mathrm{tr} / \mathrm{m}$, 4 pales orientables.

2 Coque liće au conduit hydrautique par 12 bras (avantdirectrices).

312 avant-directrices.

424 directrices mobiles.

6 Alternateur.

- Accès et sortie des câbles électriques.

des deux primaires du transformateur cotrespondant. Il existe donc 6 ensembles et 3 cransformateurs.

Leurs principales caractéristigues.

- type bulbe à axe horizontal,

- entraînement par turbine Kaplan horizontale à 4 pales à inclinaison variable,

- diamètre de la roue : $5,35 \mathrm{~m}$,

- vitesse de rotation normale de $93,75 \mathrm{tr} / \mathrm{min}$ - survitesse maximale $260 \mathrm{ir} / \mathrm{min}$, 
- ventilation en circuit fermé, le fuide de réfrigération étant de l'air surpressé à 2 bers atbsolus.

Lesr fonctionnement particulier :

Les groupes ont un fonctionnement spécifique à l'usine de La Rance, adapté aux problèmes de gestion de l'eau disponible lors des différentes phases de marée.

Ils sont réversibles mécaniquement atin de pottvoir tourner dans un sens ou dans l'autre seton le sens d'écoulement de l'eau.

Its sont réversibles électriquement afin de pouvoir fonctionner soit en générateur (turbinage) soit en moteur (pompage).

Ils dojvent en outre pouvoir servir d'orifice de passage d'eau avec deux sens possibles.

Le point cssentiel et particulier du fonctionnement des groupes bulbes de La Rance réside donc dans la réversibilité du sens de rotation tant en altenateur qu'en moteur.

Cette réversibilité est un paramètre très important et très intluent qu'il faut nécessairement prendre en compte dans la conception des éléments du butbe et dans la conception des liaisons mécaniques entre ces éléments, en particutier en ce qui concerne les alternateurs/moteurs des groupes.

\section{I LES ALTERNATELRS}

Compte tenu de ce qui est dit précédemment, is scrait logique, pour être complet, d'écrire «altemateurs/moteurs » puisque la réversibilité du transfert d'énergic existe, et ceci pour deux sens de rotation mais «alternateur" suffira pour la suite de ce résumé car dans les faits, les bulbes de La Rance fonctionnent bien plus souvent en alternateur qu'an moteur avec, pour environ les $2 / 3$ du temps, un écoulement d'eau dans le sens Estuaire $\rightarrow$ Mer.

\subsection{Description générale et quelquues dimensions}

Ce sont des machines synchrones, ce gui signifie que leur vitesse de rotation est en permanence égale à celle de ta vitesse du champ magnétiquc tournant. Ils sont de type «à pôles saillants ».

La partie fixe de l'atcrnateur, ou stator, ou induit est constituée d'un circuit magnétique de forme torique en tôle feuilletée équipé d'un bobinage triphasé à barres. Le stator cst logé dans la carcasse, celte-ci faisam partie intégrante de l'enceinte du butbe.

Cet ensemble, composé du stator complet ct de la carcasse, a une masse voisine de 35 tonnes.

La partie toumante, ou rotor, est un inducteur comportant 64 pôles sajllants dont la masse marnétique est équipée d'une bobine inductrice de 8 spires épaisses permeltant le passage d'un courant continu d'excitation voisin de $1150 \mathrm{~A}$.

Les masses polaires, en tôles magnétiques feuilletécs, sont fixées à la jante par 8 goujons traversant celle-ci et écrous freinés.

Cet ensemble, composé des pôles inđucteurs complets el de la jantc, a une masse voisine de 20 tonnes (hors croisitlon de l'arbre).

Les dimensions, hors tout, des principaux éléments sont: Circuit magrétique

stator $: \varnothing$ extérieur $=4230 \mathrm{~mm}-\not$ alésage $=3853$ $\mathrm{mm}$ - Longucur axiale de l'cmpilage $=935 \mathrm{~mm}$

Carcasse $: \varnothing$ extérieur $=4367 \mathrm{~mm}$ - Longueur $=2000$ $\mathrm{mm}-$ Epaisseur de la virole $=70 \mathrm{~mm}$ environ

Rotor $: \varnothing$ extéricur $=3844 \mathrm{~mm}-$ Entrefer $=4,5 \mathrm{~mm}$ - Longueur axiale des pôles $=935 \mathrm{~mm}$

Jante

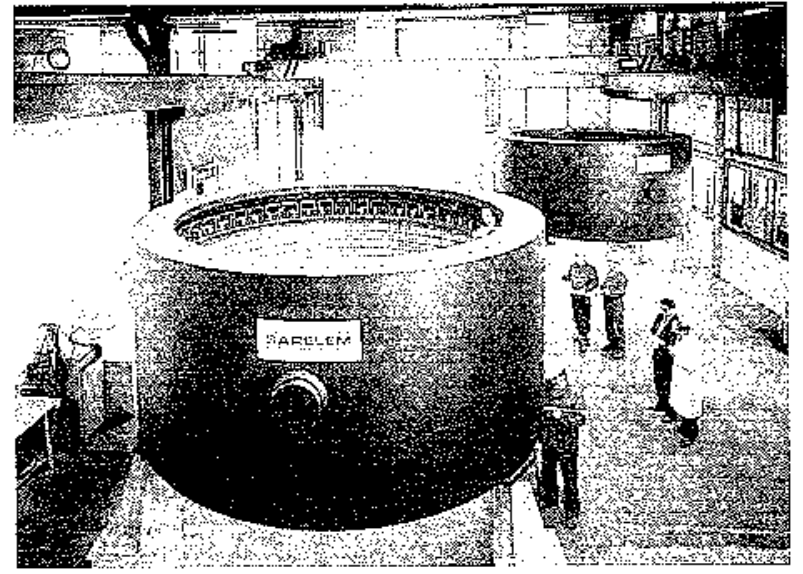

3.2 Leur principe de refroidissement, évacuation des
pertes

La transformation d'ênergie, qu'elle soit dans le sens hydraulique $\rightarrow$ électrique (alternateur) ou dans te sens ćlectrique $\rightarrow$ mécarique (moteur) ne se fat maheureusement pas sans générer des pertes diverses

- dans le cuivre,

- dans le fer,

- dans 1'air interne (ventilation),

- dans les paliers (frottements) etc.

Ces pertes doivent être évacuésis en permanence pour assurer unc stabilisation thermique dans les differents composants de l'alternateur à des températures respectant les limites imposées par le cahier des charges.

Comme pour tous les bulbes, l'alternateur des bulbes de Ia Rance, pour évacuer ses pertes, bénéficie de deux sourcès de refroidissement distinctes:

- Un circuit d'air de ventilation interne, de type axial, la pression absolue à l'intérieur de l'enceinte étant de 2 barts. Ce circuit d'air est produrt par un moto-ventilatetr placé dans l'enceinte dans l'axe longitudinal du bulbe dans le nez. côté bassin.

L'air frais est propulsé dans te croisitlon du rotor, vers le nc\% côté mer (côté hélice) d'où il cst renvoyé vers le motoventilateur. Dans ce circuit de retour le débit d'air total se partage en trois débits différents:

- un débit pour le stator passant dans des canaux axiaux situés dans les dents du circuit magnétique,

- un débit dans l'entrefer pour le refroidissement de l'alésage stator et de la surface des pôles inducteurs,

-. un đébii entre les pôles inducteurs pour refroidir le reste du rotor.

L'air chaud est refroidi par échange thermique dans un échangeur placé sur la paroi du bulbe côté bassin.

- L'eau quj transite dans la conduitc forcée du bulbe et quj évacue des pertes par convection forcéc dans l'ean à la sujface extérieure de la carcasse.

Les caractéristiques nominales sont les survantes:

Tension aux bornes (UN)

Courant stator nominal (IN)

Factcur de puisience (Cos o N)

Nombre de pôles -.. Vitesse rotation (VN)

$: 3,5 \mathrm{kV}$

$: 1650 \mathrm{~A}$

$: 0,99$

: 64-93, 75

ir/min

Courant rotor at nominal (JN)

$: 1150 \mathrm{~A}$

- en Alternateur

Puissance apparente nominale aux bornes (SN) : 10 MVA

Puissance active nominale fournie (PN) $\quad: 9,9 \mathrm{MW}$

Putssance réactive nominale fournic (QN) $\quad: 1,4:$ V var

- en Moteur

Puissance mécantique tournie ( $\mathrm{P}^{\prime} \mathrm{N}$ )

$10 \mathrm{MW}$ 


\section{1 LES ALTERNATELRS EN EXPLOITATION}

1966

La réalisation des atternateurs est effectuée pur trois constructeurs électromécaniciens :

- Alsthom qui réalise 12 illternateurs, sojit la moitić du parc instatlé.

- Jeumoni et SW qui font 6 alternateurs chacun.

\section{6-1967}

Le premier groupe est couplé sur le réseau en toût 1966. la mise en service du dernier groupe a lieu en décembre 1967.

1976

Le premier stator doit être reçonstruit pour raison principale tiée à jut détérioration de son circuit magnétique statorique : rupture de clavetes d'mpilage, desserrage longitudinal, etc..

\section{6 à 1982}

Tous les stators sont reconstruts pour des raisons liées directement à la détérionation des circuits magnétiques

- Alsthom reconstruit le premier stator, celui du B5, en 1976.

- LK reconstruit 17 stators entre 1977 et 1982.

- Repelec reconstruil 6 stators entre 1977 et 1984.

\section{5}

Malgré dimportantes améliorations de conceptions et de réalisations apportées lors de la première reconstruction au niveau du circuit magnétique statorique (fixation à la carcasse et serrage longitudinal), les exploitants décident de fairc reconstruire à nouveau certains stators.

\section{5-1996}

La Société SARELEM, filiale du groupe Framatome est retenue par E.D.F. pour reconslruire 7 stators el construire 2 stators neufs tous interchangeables. La rcconstruction du B5 et celle du premier rechange scront achevées lin 1996.

\section{DA RÉNOVATION EN COURS DES ALTERNATEURS DE LA RANCE}

Les problèmes de fuabilité survenus après la construction initiale et après la première reconstruction des stators, ont pour origine principale le circuit magnétique.

Le mode de fonctionnement particulier et contraignant des groupes dc $\mathrm{E}$ a Rance un facteur prédominant dans le processus d'usure relativement rapide du circuit magnétigue et i convient, avec les autres paramètres, de le prendre cn considération dans une nouvelle conception et réalisation.

Dans un plan de coupe perpendiculare à l'axe longitudinal du groupe, on trouve un composant dont le rôle est essentiel dans le circuit magnétique : le secteur élémentaire de tôle magnétique, pièce de base de l'empilage

Ce secteur est un morceau de tôle en segment angulaire détimité à l'extérieur par un contour grlobalement cylindriquo dans lequel figurent les formes en trapèze destinćes à recevoir lo clavetage liant le secteur à ta carcasse. Son contout intérieur est également cylinđrique et correspond au diamètre de l'alésage. Ce contour comporte les encoches destinées à recevoir les bases da bobinage.

Les efforts exercés sur ce sccteur dans le plan de coupe :

a) Les groupes de La Rancc sont réversibles, ils peuvent tourner dans un sens ou dans l'autre en alternateur et en motcur. Le couple électromagnétique induit des efforts tangentiels sur le diamètre extérieur du secteur, Ces efforts sont repris par le clavetage du secteur sur la carcasse.
Pour éviter des déplacements angulaires du secteur par rapport au clavelage, il faul, comple łcnu de la réversibilité des efforts, que le clavelage de fixation soit insensibte au sens de rotation, done au sens des efforts. Cette insensibilité aux efforts alternés doit être obtenue par une conception du clavetage telte que te secteur soit bloqué angujtirement en un point de fixation sans jeu.

Sans cette disposition, is chachere changement du sens do rotation, le secteur risquerait de se déplacer angulairement de la valeur du jeu, ce qui tinirait par le mater al droit du clavetage, puis it prendrait davantage de jeu par fatigue cumulée etc... jusqu"ì se dégrader inexorablement. Le secteur doit donc être calé, sans jeu, angulairement en un point de tixation.

Simultanément. le secteur est soumis à des contraintes thermiques dues à la température. l] doit pouvoir assurer une dilatation angulaire, done posséder des jeux de montage fonctionnels dans te sens angulaire.

b) Le secteur de tôle magnétique est soumis également aux efforts d'utlraction magnétique qui naissent au niveat de I'entrefer par création de pôles magnétiques de signes contraires (Nord-Sud) qui sateirent. Cet elfort radial doit être repris par le clavetage extéricur entre le secteur et ta carcasse.

Cet effort ast pulsaloire avec une fréquence égate àt la fréquence de rotation multipliée par le nombre de pôles.

La fréquence de rowation $F$ rétant égale à $F / p=$ fréquence du résea (50 Hz) et $p$ le nombre de paires de pôles inducteuri, Ia fréquence pulsatoige a donc pour valeur:

$$
F_{\mu}=F_{r} \times 2 p=2 F \text { soil } 100 \mathrm{H} \%
$$

L'axe principal du groupe étant horizontal il convient d'ajouter l'effort dû au poids des tôles magnétiques avec le bobinage ramené au cluvelagé.

c) Dans un plan de coupe longiludinal, le circuil magnétique feuilleté ext composé de secteurs empilés coupés dans leur epaisseur, Le nombre de secteurs empilés est proportionnel à la longueut du circuit magnétique.

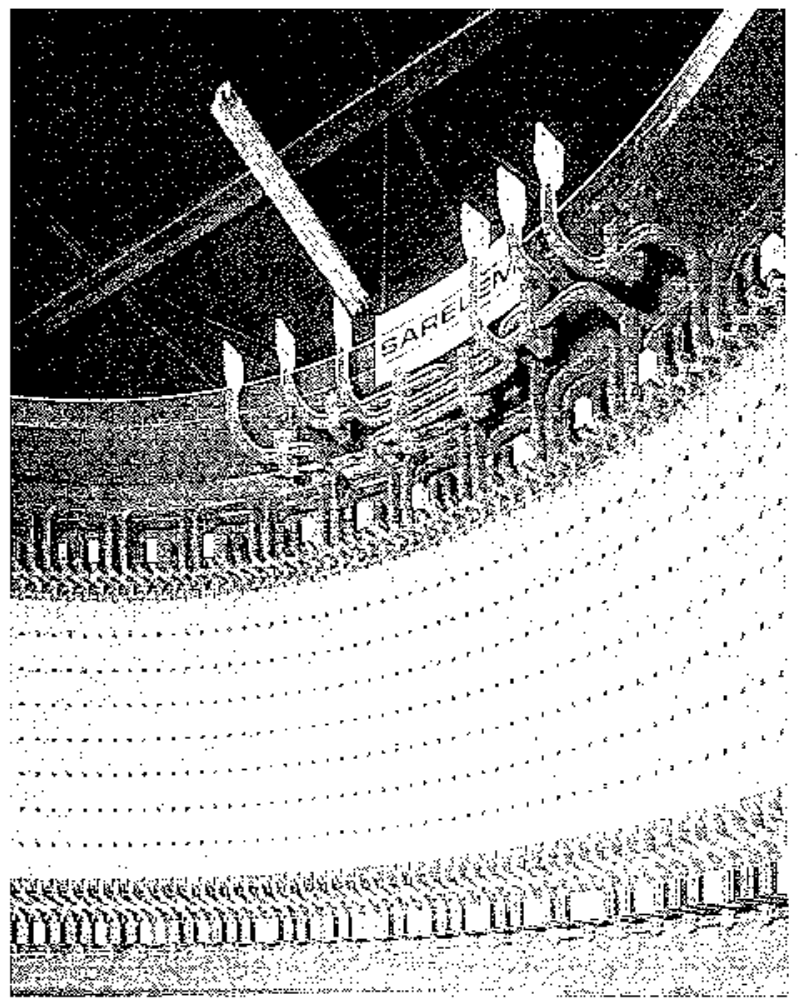


Sur cet axe iongitudinal, le circuit magnétigue doit être maintenu serté cn permanence, quel que soit le type de fonctionnement, en régime stabilisé ou en régime transitoirc (phase đàrêt on phase de démamute).

Le serrage tongitudinal pemanent doit être assuré par un systène suffisamment souple et possédant une bonne élasticite de façon is ce que la dilatation thermique longitudinale du circuit magnétique soit possible en fonctionnement el que celui-ci puisse reprendre sa longueur intiale lors d'un retour à l'état froid après arrêt đu groupe.

On dit communément que le circuit magnétique doit être « libre de rẹspirer " sans se desserrer dans te temps.

di) Le circuit magnétique composé de sectetrs empilés est sounis axialement anx efforts de dilatation et itux efforts magnétiques. Ces contraintes induisent dans te temps un phémomène de tassement des tôles qui condut obligatoirement à un desserrage relatif longitudinal de l'empilage.

Il faut donc concevoir à l'origine, un système pernetant le resserrage axial éventuel du circuil magnétique en période d'exploitation. La place dispunible n'étant pas importante dans un bulbe, il est nécessaje que le système de resserrage puisse permettre physiquement une accessibilitć sudtisante à l'operation.

Il était donc fondamental que la nouvelfe reconstruction des stators soit basée sur une étude technique de conception approfondie et détaillée prenźnt en compte ces purrémètres importants dus aux contraintes de fonctionnement spécifique et contraignant des bulbes de La Rance, ceci principalement pour la partie relative au circult magnétique et compte tent des problèmes rencontrés après les constractions précédentes.

Celte nouvelle éfude a d'allleurs débuté par une andlyse des conceptions précédentes, retenant ce qui était bon et fiable en l'amétiorant par nombre de détails techniques et technologiques.

\subsection{Renouveau du cireuit magnétique}

Pow sa conception générule et ses détails, i] s'inspire d'une conception existante dite «à tiges de serrages traversantes ».

Le sectetur magnétique élémentaire est en tôle magnétique de faible ćpaisseur $(0,5 \mathrm{~mm})$ laminée à froid don les caractéristiques sont à cycle d'hystéris étroit avec un niveau do pertes fer (par hystéris et courants de Foucauli) très bas : $1,1 \mathrm{~W} / \mathrm{kg}$ aे 1 Jesla at $50 \mathrm{H} \%$.

Ce secteur élémentare est découpé par matriçağe en une seute opération à partir d'une bande de tôle cn roulcatu prétzlablement isolée sur ses deux faces par un vornis phénolique de classe $H$. Après matriçage, le contour du secteur n'est pas ébavuré afin d'ćvitcr de détériorer l'isolation.

La hauteur de la bavure a une valeur maximum imposée de $2 / 500^{2}$ de mm, son contrôle est effectué chè le découpeur.

Le profil exlérieur du secteur comporte dans sa partie centrale une découpe en forme de queue d'aronde mâle qui prendra place cntre deux claveites de section trapézoidate (trapèze rectangle) dites «en marteau », fixées à la carcasse par des vis à tête six pans creux à haute résistance mécánique.

Le profil intérieu du secteur (côté alésage) comporte 8 rainures ou encoches destinées à recevoir les bares du bobinage après cmpilagc.

Lors de l'opération d'empilage, les sccteurs sont placés dos à dos, ou à retournement, afin de les places bavure contre bavure. L'isolation du feujlletage de l'empilage est done obtenue enlre chaque lit élémentaire constitué de deux tôles dos à dos

A l'empilage, les lits de tôles élémentaires sont décalés de la valeur d'un demi-secteur, soil au demi-recouvrement. Cet enchevêtrement d'un demi-pas de secteur permet d'obtenir une bome rigidité mécanique du tore magnétique.
Radiatement, chaque secteur est done tert en son axe méöian par deux claveltes trapézoödales (trapèze rectangle) réglées avant montage pour avoir un serrage important str la clavette mâle centrale du secteur.

Le circuit magnétique du stator comporte 240 encoches: it faut done 30 secteurs de 8 encoches pour effectuer un tour d'empilage. Ce tontr nécessite 2 clavettes par secteur, soit au total 60 clevettes par cour.

L'empilage étant au demi-recouvrement, it faut donc au total 2 fois 60 clavettes, soit 120 clavettes pour l'ensemble de l'empilage. Chaque pare de clavettes tenant un secteur est donc expacée régatièrement angulairement de $3^{2}$.

Le réglage des claveltes dans l'espace doit done être parfait (tolérance dars chaque dimension maximum admise $1 / 10^{\circ}$ de mm) atin d'obtenir un tore málgnétique possédant des jeux fonctionneis internes égaux et réguliers de l'ordre te $3 / 10^{\circ}$ de nm. Ces jeux atutoriseront les mouvements normaux du circuit magnétique soumis aux efforts liés aux contraintes de fonctionnenjent : mécaniques, mugnétiques et thermigues.

54000 secteurs élémentaires scront empilés dans la carcasse pour constituer le circuit magnétique.

Outre le contour extéricur avec claveltes et le contour intérieur avec denture, chaque secteur comporte des découpes supplémentaires:

- 4 urous dans la couronne pour passage des liges de serrage axial (tiges traversuntes),

- 16 trous en forme de bontonnière (2 par dents) pour permettre le pasisalge de l'air de ventilation.

\section{I.I Dispositif de serrage axial du cirent magnétique}

Il est du type à liges traversintes, l'effort de sergage étant réparti str les faces du tore par ues piaques de scrrage, or pavés. Chaque lige de serrage est plicée dans un tube en matériau isolant afin d'éviter des courants de circulation et des pertes supplémentajes dars les tiges dues au passage du cramp magnétique tournant.

Outre les tiges, l'ensemble du système de serrage est isolé clectriquement du circuit magnétique, par un système de rondelles isolantes placées entre les écrous de serrage.

Chaque secteur magnétique comporte 4 liges de serrage soit au total $\varsigma \times 30=120$ liges de serrage, pour l'ensentele du tore, avec un dispositif isolant pour chaque tige.

Un dispositif particulier et original, prévu pour chaque tige, permet de reporter, côté mer (côté opposé aux connexions du botinage) le point d'appui sur les pavés de serrage à l'cxtérieur du bobinage alin de factiter !'aceessibjlicé pour resserrage éventucl en cours d'exploitation, l'outillage nécessaire à cette opération étan fourni avec le stator.

\subsubsection{Les tiges de serrage axiules du circuit magnétique}

Eltes sont réalisées dans un acier nickeluchrome, à haule résistance mécanique, étirê à froid $R e \geq 700 \mathrm{MPa}$. Chaque extrémité conporte un filetage à pas fin réalisé par moletage afin d'ćviter de couper les fibres du métal.

Ce filletage formé el non usiné est conique extérieurement (pente $6 \%$ ) ain de faire travailler tous les filets en prise sur l'écrou de la même façon contrairement à un fitetáge cylindrique dont la probabilité est de faire travailler davantage les premicrs filets en prise que les demiers.

\subsubsection{Les pavés de serrage.}

Ils sont réalisćs dans un altiage d’afuminium (FORTAL) à caractéristiques mécaniques comparables à celles a"un acier 
E 36. Le pavé est obtenu par usinage dans la masse an $\mathrm{CN}$. Chaque pavé comporte 4 encoches; il faut donc 60 pavés par côté de tore, soic 120 pavés pour l'ersemble du circuit magnétique.

\section{I.4 Montage da circuit magrétique .. Aspect technique er sechnologigue}

Les opérations de réglage et de fixation des clavettes, de réglages au démarrage du circuit magnétique, de montage du circuit magnétique avec passages intermédiaires maintenus jusqu'à la f̂́n de !'opération nécessitent un nombre de détails technologiques importants spécifiques aux reconstructeurs qui font l'objet principal de l'exposé.

Touss ces détuils ne peuvent être repris dans un résumé. ll en est de même pour les opérations de serrage final par tension des tiges de serrage, à l'aide d'un système actif par vérins creux, apłès l'essai de magnétisation.

\section{I.5 Le comportement de l'enpilage pendan les phases de fonctionnement}

Les contruntes essentieltes qui agissent sur le circuit magnétique sont importantes pentant les phases transitoires: at démarrage et à l'arêt du groupc.

Elles influent, par fatigue cumulée, sur la tenuc dans le temps du circult magnétioue. Certains phénonienes ne sont pas totalement compensés par l'uspect conception. Il en est ainsi du phénomène de tassement axial relatif du circuit magnétique dô aux contraintes magñétiques et thermiques agissant pendant le fonctionnement. Pour cela, SARELEM a imaginé un systeme de resserrage original accessible permettant de reprendre le resserrage des circuits magnétiques en exploitation.

\subsection{Le nouveau bobinage}

Conçu pour les 240 encoches du circuit magnétique, le bobinige est du type "ondulé à barres». Son couplage est róalisé en trịhasé - étoile - série.

Il est à deux faisceaux ou barres par encoche soit 480 barres pour l'ensemble du bobinage monté.

Chaque barre est composée de brins de cuivre électrolyticue de section rectangulaire formés et tressés entre eux pour assurer une compensation de champ transversal d'encoche selon procédé Roëbel.

L'isolation des barres est réalisée avec un ruban micacé posé par enrubannage continu sur machine automatique. 正 mur isolant de masse obtenu est ensuite imprégné en cuve sours vide poussé avec une résine Epoxy de classe électrothermique $F$.

La polymérisation de la résine est réalisée en étuve pilotée selon un cycle de température contrôlé, les barres ćtant montées dans un outillage de conformation rigide.

En finition, avant essais diélectriques, ta partic frotte des barres est recouverte d'un vernis conducteur permettart d'obtenir une électrode de masse de bonne qualité destinée à éviter le phénomène de décharges partielles dans les encoches.

Un vernis semi-conducteur autorise l'écoutement des charges électriques, naissant dans les développantes, vers l'électrode de masse.

Le calage radia d'encoches est réalisé par un procédé spécial avec calage souple appelé « Ripple Spring agissant comme un ressort précontraint.

La précontrainte est obtenue par un écrasement de $80 \%$ du ressor à l’aide de deux demi-cales biaises.

Ce système de précontránte sur les barres dans l'encoche, permet d'ćviter le dessenage du calage dû an tassement dan je temps des éléments composant les barres qui sont sou. mises aux efforts électro-dynamiques crés par le champ transwersal d'encoches. 